\title{
Glückliche Sklaven
}

Während sich der von der Safe-Harbor-Entscheidung des EuGH vom 06.10.2015 aufgewirbelte Staub langsam legt, die Aufsichtsbehörden erste Bußgeld-Bescheide verschicken und internationale Unternehmen beginnen, die von der EU-Kommission in Eile gezimmerte und am 12.07.2016 verabschiedete neue Rechtsgrundlage für die Übermittlung personenbezogener Daten in die USA - das EU-US Privacy Shield-Abkommen - umzusetzen, erreicht die heimliche Überwachung neue Dimensionen.

Anfang der 2000er Jahre schossen sich Datenschützer auf die Nutzung von Cookies als Tracking-Instrument ein. Darauf reagierte die EU am 25.11.2009 mit der Verabschiedung einer (in diesem Punkt wenig Technik neutralen) "Cookie-Richtlinie“, die unter anderem die Nutzung von Cookies an eine Einwilligung der Benutzer bindet. Sie ist bis heute in vielen EU-Staaten nicht umgesetzt; Verstöße werden von den nationalen Datenschutzaufsichtsbehörden nicht geahndet. Neben den praktischen Schwierigkeiten, die sich bei der technischen Realisierung einer Cookie-Einwilligung ergeben, hat sich die Regelung allerdings auch inhaltlich überlebt: Cookies sind schon lange nicht mehr der Tracking-Mechanismus der Wahl. Statt über dezentrale Datenkrümel (die sich immerhin löschen lassen) werden Web-Nutzer heute via "Browser Fingerprint" und IP-Adresse identifiziert.

Derweil weichen Google \& Co. auf andere, weit ergiebigere Methoden zur Gewinnung von Internet-Nutzungsdaten aus: Mit "kostenlosen“ Service-Angeboten spannen sie seit Jahren Webseitenbetreiber vor ihren Karren. Mit Erfolg: Das WebseitenAnalysetool Google Analytics erreichte einen Marktanteil von über $90 \%$, bevor es in den Fokus von Datenschützern geriet. Der Kompromiss zwischen Datenschutz-Aufsicht und Google, die Erhebung und Verarbeitung der Daten durch Google als „Auftragsdatenverarbeitung" zu gestalten und die Option eines "Opt-Out-Cookies" anzubieten, war datenschutzrechtlich schon immer eigenwillig und hat Google Analytics in Deutschland einen stabilen Marktanteil von über $50 \%$ bewahrt. Inzwischen wird die Vereinbarung im Lichte des EuGH-Urteils zu Safe Harbor von den Aufsichtsbehörden immerhin seit Monaten "geprüft".

Während sich die Aufsichtsbehörden an Google Analytics und Social Media Plugins (wie dem „Like Button“ von Facebook) festbissen, zündeten Google \& Co. ein Feuerwerk weiterer Service-Angebote für Webentwickler, die diese bereitwillig in ihren Code einbetteten. Inzwischen gibt es kaum noch eine Webseite, die kein Javascript- oder CSS-Framework und keinen Web-Font nachlädt - und dabei jedes Mal, als heimlicher Nebeneffekt, die Nutzerdaten bei Facebook, Google oder Twitter abliefert.

Schließlich gelang Google mit der Safe Browsing-API der ultimative Coup: Einmal in den Browser integriert, prüft sie jeden Webseitenaufruf gegen eine Google-Blacklist auf enthaltene Schadsoftware. Inzwischen ist die API Teil von Firefox, Safari und Chrome - und schickt jede zweifelhafte der von einer Milliarde Web-Nutzern ausgewählten Web-Adressen (URLs) inklusive geeigneter Identifikationsdaten (IP-Adresse oder "Browser Fingerprint") an Google. Da die URLs nicht gehasht werden, erhält Google so ein umfangreiches Internet-Nutzungsprofil, das mit Daten der Suchmaschine, Google+, GMail, Youtube und Google Analytics angereichert wird. Da wollte auch Microsoft nicht zurückstehen - und führte im IE8 den SmartScreen-Filter ein.

Sicherheit für den Preis einer (mehr oder weniger) freiwilligen Profilpreisgabe: Ein genialer Schachzug. Denn die erbittertsten Feinde der Freiheit sind bekanntlich die glücklichen Sklaven.

Mit herzlichen Grüßen, Ihr

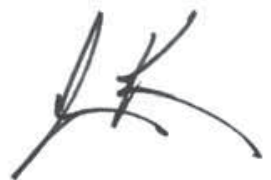

Dirk Fox 